\title{
LES PARCOURS SCOLAIRES DES JEUNES CANADIENS D'ORIGINE IMMIGRANTE AUX ÉTUDES SUPÉRIEURES : QUI Y ACCÈDE ET COMMENT?*
}

\author{
Pierre Canisius Kamanzi ${ }^{1}$ (D)
}

RÉSUMÉ: Cet article vise à examiner dans quelle mesure les jeunes Canadiens d'origine immigrante font usage des parcours linéaires et non linéaires aux études supérieures. Les résultats obtenus à partir des données tirées du fichier public du ministère de l'Éducation du Québec permettent de dégager les constats suivants. Premièrement, ces jeunes poursuivent les études supérieures par la voie des parcours linéaires dans une proportion plus élevée que leurs pairs dont les parents sont nés au Canada. Deuxièmement, les différences sont nettement plus élevées à l'université qu'au CEGEP ${ }^{1}$. Troisièmement, il existe des différences significatives importantes parmi les jeunes d'origine immigrante euxmêmes selon l'origine géographique des parents et selon que l'étudiant est un immigrant de $1^{\mathrm{e}}$ ou de $2^{\mathrm{e}}$ génération.

Mots-clés: Enseignement supérieur. Parcours scolaires. Inégalités scolaires. Immigrants. Canada.

\section{OS CAMINHOS AO ENSINO SUPERIOR DE ESTUDANTES DE ORIGEM IMIGRANTE NO CANADÁ: QUEM OS REALIZA E COMO?}

RESUMO: $\mathrm{O}$ artigo visa examinar até que ponto os jovens Canadenses de origem imigrante fazem uso de caminhos lineares e não lineares ao ensino superior. Os resultados obtidos a partir dos dados do arquivo público do Ministério de Educação de Quebec permitem tirar as seguintes conclusôes. Em primeiro lugar, esses jovens buscam o ensino superior através de caminhos lineares em maior proporção do que seus pares cujos pais nasceram no Canadá. Em segundo lugar, as diferenças são significativamente maiores na universidade do que no CEGEP. Em terceiro lugar, existem importantes diferenças entre os jovens de origem imigrante de acordo com a origem geográfica dos pais, e se o estudante é um imigrante de primeira ou de segunda geração.

Palavras-chave: Ensino superior. Percursos escolares. Desigualdades escolares. Imigrantes. Canadá.

\footnotetext{
*Projet de Recherche. Organisme subventionnaire: Conseil de recherche en sciences humaine du Canada (CRSH) /Social Sciences and Humanities Research Council of Canada (SSHRC).

${ }^{1}$ Université de Montreal - Montreal, Canadá. E-mail: pierre.canisius.kamanzi@umontreal.ca DOI: 10.1590/ES0101-73302019181525
} 


\title{
THE HIGHER EDUCATION PATHWAYS OF CANADIAN IMMIGRANTS STUDENT: WHO TRAILS THEM AND HOW ?
}

\begin{abstract}
This article aims to examine to what extent Canadian youth of immigrant origins pursue higher education through linear or non-linear pathways. The data used in this article come from the public administrative file of the Quebec Ministry of Education, and three conclusions can be drawn from results. Firstly, these youth pursue higher education via linear pathways at a higher proportion than their peers whose parents were born in Canada. Secondly, differences are significantly higher at the university level than at the CEGEP. Thirdly, there are significant differences among youth from immigrant backgrounds specifically. Access to higher education and the undertaking of linear educational pathways vary according to parental geographic origin and whether the student is a first- or second-generation immigrant.
\end{abstract}

Keywords: Higher education. Academic pathways. Educational inequalities. Immigrants. Canada.

\section{LOS CAMINOS A LA ENSEÑANZA SUPERIOR DE ESTUDIANTES DE ORIGEN INMIGRANTE EN CANADÁ: ¿QUIÉN LOS REALIZA Y CÓMO?}

RESUMEN: El artículo pretende examinar hasta qué punto los jóvenes canadienses de origen inmigrante hacen uso de caminos lineales y no lineales a la enseñanza superior. Los resultados obtenidos a partir de los datos del archivo público del Ministerio de Educación de Quebec permiten sacar las siguientes conclusiones. En primer lugar, esos jóvenes buscan la enseńanza superior a través de caminos lineales en mayor proporción de que sus pares cuyos padres nacieron en Canadá. En segundo lugar, las diferencias son significativamente mayores en la universidad de que en el CEGEP. En tercer lugar, existen importantes diferencias entre los jóvenes de origen inmigrante de acuerdo con el origen geográfico de los padres, y si el estudiante es un inmigrante de primera o de segunda generación.

Palabras clave: Enseńanza superior. Recorridos escolares. Desigualdades escolares. Inmigrantes. Canadá.

\section{Introduction}

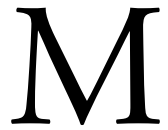

algré l'égalité des chances d'accès à l'école dans les pays développés, plusieurs études témoignent des inégalités scolaires persistantes entre les élèves issus des familles immigrées et leurs pairs dont les parents sont natifs du pays d'accueil. Les premiers ont des résultats scolaires relativement plus faibles par rapport aux seconds en ce qui a trait à l'enseignement de base (primaire et secondaire) (DUSTMANN ; FRATTINI ; LANZARA, 2011). 
Cette situation est particulièrement observée dans les pays de l'Europe continentale, comparativement aux pays anglo-saxons où les différences semblent plutôt modérées (DUSTMANN ; FRATTINI ; LANZARA, 2011), ce qui appuie ainsi l'hypothèse des effets de ségrégation des politiques publiques et institutionnelles. En outre, bien qu'une grande partie arrive à terminer la scolarité secondaire en raison de son caractère obligatoire, ces jeunes sont vite mis au défi par la sélection au secondaire qui freine leurs ambitions scolaires (BAUM \& FLORES, 2011 ; GRIGA \& HADJAR, 2013). La sélection précoce dans certains pays est telle que seule une petite minorité est admise dans des filières ou programmes de formation générale conduisant à l'enseignement supérieur, tandis que la majorité est affectée dans des filières professionnelles de type court conduisant au marché du travail et la contraignant à mettre fin à la scolarité (CRUL et al., 2012).

Afin d'atténuer cette forme de ségrégation qui affecte davantage les élèves de milieux défavorisés dont fait partie la grande majorité des minorités ethnoculturelles issues de l'immigration, plusieurs pays ont mis en place des mesures visant à assouplir les conditions d'admission aux études supérieures. Ces mesures visent à diversifier les parcours scolaires conduisant à l'enseignement supérieur, notamment par une meilleure articulation avec l'enseignement secondaire professionnel. Leur objectif consiste à élargir l'accessibilité des études supérieures et à répondre davantage à la demande de main-d'œuvre hautement qualifiée de plus en plus exprimée par les entreprises (HOELSCHER et al., 2008; MACLEAN \& PAVLOVA, 2013). Ce réaménagement de la structure a donné lieu à des parcours non linéaires vers l'enseignement supérieur, caractérisés par bifurcations combinant souvent des programmes de formation professionnelle et générale.

Au Canada, bien qu'en moyenne, le niveau des performances scolaires des élèves d'origine immigrante et le taux de participation aux études supérieures soient comparables à ceux des autres jeunes Canadiens, cette observation est loin d'être généralisable à toutes les communautés immigrées dont certaines affichent encore des taux faibles de persévérance scolaire et d'accès aux études supérieures (MC ANDREW, 2015 ; THIESSEN, 2009).

La présente étude vise à examiner dans quelle mesure les jeunes Canadiens d'origine immigrante résidant dans la ville de Montréal (province du Québec) font usage de ces différents parcours linéaires et non linéaires. Autrement dit, dans quelle mesure leurs parcours à l'enseignement supérieur se comparent-ils à ceux de leurs pairs non immigrants? Le texte est divisé en quatre sections. La première fait un bref aperçu des écrits sur les facteurs à l'origine des inégalités scolaires entre les immigrants et les autres élèves ou étudiants. La deuxième décrit la méthodologie : les données utilisées, les variables étudiées et les stratégies d'analyse, alors que la troisième présente les résultats. Nous terminons par une conclusion qui résume les principaux constats et propose quelques pistes interprétatives. 


\section{Parcours scolaires des immigrants : entre assimilation et intégration}

Selon la littérature existante, trois hypothèses principales sont soutenues pour expliquer les inégalités scolaires entre les immigrants et les non-immigrants. La première a trait aux différences culturelles entre le pays d'origine et le pays d'accueil, la seconde aux caractéristiques des élèves et de leurs parents et la troisième au contexte de la société d'accueil.

\section{La théorie de l'assimilation}

Selon les tenants de la théorie de l'assimilation, la réussite scolaire des jeunes d'origine immigrante est tributaire du degré d'intégration des valeurs culturelles de la majorité dominante du pays d'accueil. Toute société étant stratifiée, trois principaux scénarios tendent à se produire : les immigrants s'intègrent soit à la culture de la classe dominante, soit à celle de la classe populaire ou alors ils enferment dans leurs communautés (PORTES \& ZHOU, 1993). Il s'agit d'une assimilation segmentée (PORTES ; FERNÁNDEZ-KELLY ; HALLER, 2005 ; ZHOU, 1997) qui, selon le degré de proximité ou d'éloignement par rapport à la classe dominante, détermine les chances de mobilité sociale et de bien-être (ALBA \& NEE, 1997; GREENMAN \& XIE, 2008; SILBERMAN ; ALBA ; FOURNIER, 2006).

Dès lors, l'intégration et la mobilité sociale sont d'autant plus faibles qu'une communauté issue de l'immigration entretient des rapports d'évitement avec la classe dominante et vit en exclusion par rapport au reste de la société et vice versa. Or, dans un même pays d'accueil, les rapports de proximité ou d'évitement varient fortement entre communautés immigrantes (PORTES \& ZHOU, 1993). Les chances d'intégration ou le risque d'exclusion vont diminuer ou accroître selon que leurs pays d'origine different ou ressemblent au pays d'accueil sur le plan socioéconomique (BORJAS, 2006), mais surtout culturel (BELOT \& EDERVEEN, 2012; LEVELS ; DRONKERS ; KRAAYKAMP, 2008). Ainsi, de manière générale, les immigrants originaires des pays européens ont des performances et des parcours scolaires comparables à ceux des natifs. Par contre, à quelques exceptions près, ceux qui viennent des pays du Sud ont des résultats scolaires plus faibles et accèdent à l'enseignement supérieur dans une proportion également faible. Des recherches révèlent que, même quand ils constituent des communautés importantes, ces derniers ont des parcours scolaires fragiles. Tel est le cas des Mexicains aux États-Unis et des Maghrébins en France (ALBA \& SILBERMAN, 2002; BRINBAUM \& LUTZ, 2017 ), ainsi que des Turcs en Allemagne (SÖHN \& ÖZCAN, 2006).

\section{Ressources des parents}

Si tous les immigrants cherchent à s'intégrer dans les structures des pays d'accueil pour améliorer leurs conditions de vie, rares sont les adultes qui parviennent à 
retrouver un statut socioéconomique équivalent à celui dont jouissaient auparavant dans leur pays d'origine. Pour la majorité d'entre eux, la scolarisation de leurs enfants constitue le principal moyen d'envisager une mobilité sociale et éventuellement de recouvrer indirectement leur propre statut socioéconomique (ICHOU, 2014). Dès lors, l'accès à l'enseignement supérieur et à ses diplômes constitue une stratégie de premier choix pour atteindre cet objectif. Il s'avère toutefois que peu d'entre eux atteignent cet objectif en raison du manque ou de l'insuffisance des ressources pour soutenir longtemps la persévérance de leurs enfants aux études (BAUM \& FLORES, 2011 ; NIELSEN et al., 2003).

Comme le plus souvent les familles immigrantes ont des revenus modestes et vivent dans une précarité économique, seuls les jeunes dont les parents sont nantis d'un capital scolaire moyen ou élevé et, dans le meilleur des cas, sont détenteurs eux-mêmes d'un diplôme d'études supérieures ont la chance de s'en sortir (BAUM \& FLORES, 2011). Le capital scolaire des parents agit sur deux axes a priori complémentaires et dont les effets sont interdépendants. D'une part, les parents plus scolarisés sont à même de transmettre à leurs enfants un capital culturel favorable au développement des habitus requis par l'intégration et la réussite scolaire (BOURDIEU, 1966). D'autre part, leur capital scolaire permet de générer le capital social favorable à la persévérance aux études.

Les recherches montrent que les enfants des familles immigrées qui réussissent à l'école se distinguent par ainsi un capital social élevé (BANKSTON, 2004). Celui-ci sert de catalyseur aux autres ressources (capital économique ou culturel) ou de facteur compensateur en cas de déficit (KAMANZI et al., 2007). Premièrement, les parents scolarisés sont mieux éclairés pour structurer le parcours scolaire de leurs enfants (HAO \& BONSTEAD-BRUNS, 1998). Deuxièmement, ils sont à même de s'impliquer dans la scolarité de leurs enfants et de négocier des choix scolaires stratégiques. Troisièmement, tous ces éléments créent auprès de leurs enfants l'intérêt et la confiance pour leur réussite.

\section{Le contexte sociétal}

Les explications fondées sur les théories de l'assimilation et des ressources s'avèrent cependant limitées en raison de leur vision unilatérale, car elles se focalisent seulement sur l'action des immigrants et de leurs communautés, mais tiennent peu ou pas compte de l'influence du contexte sociétal. En particulier, la théorie de l'assimilation ne s'applique qu'aux immigrants de première génération ${ }^{2}$ (ALBA \& NEE, 1997). Bien qu'ils soient en contact avec la culture du pays d'origine des parents et que certaines valeurs puissent constituer un obstacle réel à l'intégration dans la société d'accueil (ELDERING \& KNORTH, 1998), les jeunes immigrants de deuxième génération ${ }^{3}$ ont avant tout comme références culturelles principales celles du pays où ils sont nés et grandissent, comme le soulignent Schneider \& Crul (2012). Dans cette perspective, la théorie du contexte social que proposent ces auteurs soutient que 
les inégalités entre les immigrants et les non-immigrants sont, non seulement, le produit des politiques publiques, mais également des rapports entre les deux groupes à travers l'organisation des institutions sociales et scolaires.

Schneider \& Crul (2012) avancent deux arguments principaux pour soutenir leur théorie. Premièrement, en aval, l'intégration des immigrants est tributaire des orientations idéologiques des politiques publiques. À cet égard, les études internationales montrent que la réussite scolaire des immigrants tend à être beaucoup plus faible dans des systèmes stratifiés avec une sélection scolaire précoce (BUCHMANN \& PARRADO, 2006 ; TELTEMANN \& SCHUNCK, 2016). Il s'ensuit des disparités importantes d'accès à l'enseignement supérieur (GRIGA \& HADJAR, 2013). Deuxièmement, en amont, l'intégration des immigrants dépend de la culture et de l'organisation des institutions locales. Alors que l'immigration est essentiellement urbaine, certaines villes sont traditionnellement marquées par la ségrégation, voire la discrimination et la marginalisation ethniques. Ces rapports ont des effets sur les pratiques d'intégration institutionnelles des élèves d'origine immigrante dans les établissements, car elles influent sur les relations avec les autres élèves, le personnel enseignant, les directions d'établissement, ainsi que sur les relations entre les parents et l'école (XIE \& GREENMAN, 2011).

Dans une étude portant sur sept pays européens (Allemagne, Autriche, Pays-Bas, Belgique, Suisse, France et Suède), Crul et al. (2012) observent que les inégalités scolaires entre immigrants et non-immigrants varient fortement entre pays, mais aussi entre villes au sein d'un même pays. Autrement dit, même à l'intérieur d'un pays comme le Canada, qui valorise le multiculturalisme (BERRY \& KALIN, 1995) et où les résultats scolaires sont comparables entre les immigrants et les non-immigrants (DUSTMANN, LANZARA \& FRATTINI, 2011; HOCHSCHILD \& CROPPER, 2010), rien n'indique que la situation est similaire partout où s'installent les familles immigrantes (HENRY \& TATOR, 2009). On peut, au contraire, avancer l'hypothèse que la situation peut fortement varier entre les lieux de résidence selon les pratiques locales d'intégration. C'est dans cette perspective que la présente étude s'intéresse aux parcours scolaires à l'enseignement supérieur des jeunes issus de l'immigration résidant à Montréal et ses banlieues (province du Québec), l'une des trois villes canadiennes qui accueillent le plus d'immigrants aux côtés de Toronto (province de l'Ontario) et de Vancouver (province de la Colombie-Britannique).

\section{Méthodologie}

\section{Sources des données et échantillon}

Les données utilisées dans cet article sont tirées du fichier administratif du ministère de l'Éducation du Québec. L'échantillon est composé 20387 élèves 
dont $5334(26 \%)$ ont au moins un parent né à l'étranger (voir Tableau 1) et faisant partie de deux cohortes d'élèves entrés en première année du secondaire respectivement en 1994-1995 ( $\mathrm{n}=9485)$ et en 2002-2003 ( $\mathrm{n}=10$ 902).

En outre, la base de données contient des données longitudinales permettant d'étudier les parcours scolaires des élèves sur une période de 10 ans, ainsi que des renseignements sur certaines caractéristiques sociodémographiques, notamment le genre et le quartier de résidence des parents. En nous basant sur le lieu de résidence des parents en $1^{\mathrm{e}}$ année du secondaire, nous avons complété cette base par des données écologiques à partir des renseignements tirés du recensement canadien de 2006, en ce qui concerne notamment les caractéristiques socioéconomiques du milieu de résidence. À cet égard, deux variables écologiques intégrées seront prises en compte dans cet article: le revenu médian par ménage et le pourcentage des diplômés d'université dans la zone de résidence des parents. L'intégration de ces deux variables dans l'analyse vise à tenir compte l'influence de l'origine socioéconomique, comme nous le précisons dans la section suivante.

\section{Tableau 1}

Répartition des sujets selon l'origine géographique des parents et le type de parcours scolaire aux études supérieures (ÉS).

\begin{tabular}{l|c|c|c|c|c|c}
\hline $\begin{array}{l}\text { Pays de } \\
\text { naissance } \\
\text { des parents }\end{array}$ & $\begin{array}{c}\text { Accès aux } \\
\text { ÉS : oui }\end{array}$ & $\begin{array}{c}\text { Accès aux } \\
\text { ÉS : non }\end{array}$ & Parcours I & Parcours II & Parcours III & \multirow{2}{*}{ N } \\
\cline { 2 - 6 } Canada & 35 & 64 & $\%$ & $\%$ & $\%$ & 14594 \\
\hline $\begin{array}{l}\text { Un seul parent } \\
\text { né à l'étranger }\end{array}$ & 36 & 64 & 24 & 34 & 6 & 1614 \\
\hline Asie orientale & 20 & 80 & 20 & 58 & 2 & 202 \\
\hline $\begin{array}{l}\text { Asie du Sud- } \\
\text { Est / Îles du } \\
\text { Pacifique }\end{array}$ & 32 & 68 & 26 & 38 & 4 & 634 \\
\hline $\begin{array}{l}\text { Afrique du } \\
\text { Nord et } \\
\text { Moyen-Orient }\end{array}$ & 26 & 74 & 22 & 45 & 7 & 725 \\
\hline $\begin{array}{l}\text { Afrique } \\
\text { subsaharienne }\end{array}$ & 36 & 64 & 24 & 34 & 6 & 146 \\
\hline $\begin{array}{l}\text { Europe et pays } \\
\text { Anglo-saxons }\end{array}$ & 33 & 67 & 24 & 36 & 7 & 717 \\
\hline $\begin{array}{l}\text { Amérique latine } \\
\text { et Caraïbes }\end{array}$ & 42 & 58 & 28 & 20 & 10 & 1056 \\
\hline \begin{tabular}{l} 
Tous \\
\hline
\end{tabular} & 35 & 65 & 25 & 31 & 9 & 19688 \\
\hline
\end{tabular}

Note : parcours $I$ : linéaire secondaire $\Rightarrow C E G E P$; parcours $I I$ : linéaire secondaire $\Rightarrow C E G E P \Rightarrow$ université ; parcours III : non linéaire secondaire $\Rightarrow C E G E P$. 


\section{Définition des variables étudiées}

La variable dépendante au cour de cet article est le parcours scolaire aux études supérieures (CEGEP ou université). Elle a été mesurée par le cheminement aux études pendant et après la scolarité secondaire. La base de données utilisée permet de distinguer deux catégories de parcours : 1) linéaires et 2) non linéaires. Les parcours linéaires comprennent les élèves qui, après l'obtention d'un diplôme d'études secondaires, ont poursuivi des études supérieures. À l'inverse, les parcours non linéaires incluent ceux qui, après l'obtention de ce diplôme, se sont inscrits dans un programme parallèle ou équivalent. Plus précisément, il s'agit des élèves qui, après l'obtention d'un diplôme de formation professionnelle, ont poursuivi des études dans un programme général et vice versa et ont obtenu deux diplômes. Ces considérations permettent de distinguer quatre catégories de parcours:

- non-accès aux études supérieures : les élèves qui, après les études secondaires, ont mis fin à leur scolarité, du moins pour la période d'observation ;

- parcours linéaire terminal au CEGEP : ceux qui, après l'obtention d'un diplôme d'études secondaires en formation générale, se sont dirigés vers un CEGEP dans un programme technique ou préuniversitaire, mais n'ont pas poursuivi leurs études universitaires ;

- parcours linéaire à l'université : ceux qui, après une formation générale au secondaire, se sont inscrits dans un CEGEP et ont, par la suite, poursuivi leurs études à l'université ;

- parcours non linéaire terminal au CEGEP: ceux qui, avant de s'inscrire dans un CEGEP, ont accompli une double formation au secondaire (générale et professionnelle).

La variable indépendante est l'origine géographique des parents de l'élève ou de l'étudiant. Elle a été mesurée par le pays de naissance des deux parents. Afin d'obtenir des effectifs suffisants pour l'analyse, les pays ont été regroupés par région géographique. En nous inspirant des catégorisations proposées par THIESSEN (2009), ainsi que KAMANZI et al. (2016), les élèves ont été regroupés en huit catégories suivantes :

1. Canada $(\mathrm{n}=15053)$;

2. un seul est né à l'étranger $(\mathrm{n}=1678)$;

3. Europe et pays anglo-saxons $(\mathrm{n}=737)$;

4. Asie orientale (Chine, Corée et Japon) $(\mathrm{n}=211)$;

5. Asie du Sud-Est et Îles-du-Pacifique (Inde, Pakistan, Indonésie, etc.) $(\mathrm{n}=656)$; 
6. Afrique du Nord et Moyen-Orient $(\mathrm{n}=762)$;

7. Afrique subsaharienne $(\mathrm{n}=146)$;

8. Amérique latine et Caraïbes $(\mathrm{n}=1132)$.

Les variables de contrôle. L'analyse tient compte des caractéristiques scolaires et sociodémographiques de l'élève ou l'étudiant. En nous basant sur les données disponibles, trois variables liées à la scolarité secondaire ont été incluses dans les analyses :

- la présence ou non du code de difficulté dans le dossier scolaire de l'élève ;

- le type d'établissement secondaire fréquenté (privé ou public) ;

- l'âge d'entrée au secondaire.

De même, deux variables sociodémographiques caractérisant l'environnement socioéconomique de la circonscription administrative habitée par les parents:

- le pourcentage de diplômés d'université ;

- $\quad$ le revenu médian par ménage.

\section{Analyse statistique}

Dans un premier temps, des analyses descriptives ont été effectuées pour dégager le portrait global des parcours scolaires aux études supérieures selon la variable indépendante. Dans un deuxième temps, nous avons procédé à des analyses multinomiales (ALLISON, 2003) tenant compte des variables de contrôle décrites dans la sous-section précédente. Quatre probabilités associées aux catégories de la variable dépendante décrites précédemment seront comparées:

1. $\mathrm{p}(\mathrm{y}=0)$ : non-accès aux études supérieures ;

2. $\mathrm{p}(\mathrm{y}=1)$ : parcours linéaire terminal au CEGEP ;

3. $\quad \mathrm{p}(\mathrm{y}=2)$ : parcours linéaire à l'université ;

4. $\quad p(y=3)$ : parcours non linéaire terminal au CEGEP.

Sur le plan statistique, le but de l'analyse est d'estimer l'influence de l'origine immigrante sur la probabilité de s'inscrire dans l'un ou l'autre des parcours sous l'influence des facteurs $X_{1}, X_{2}, \ldots X_{k}$. La modélisation par la fonction logit permet ainsi de comparer la probabilité associée à chacun de ces parcours à celle de l'inverse (c'est-à-dire, ne pas accéder aux études supérieures ou $\mathrm{p}(\mathrm{y}=0))$. 


\section{Présentation des résultats}

Après les études secondaires, la majorité des jeunes Canadiens résidant poursuivent les études supérieures. Comme l'indique le tableau 1, à l'âge de 22 ans, deux tiers $(65 \%)$ des jeunes dont les parents résident dans la région de Montréal ont fréquenté, à un moment ou à un autre, un CEGEP (34\%) ou une université $(31 \%)$. Le taux de fréquentation varie cependant entre les étudiants selon l'origine géographique de leurs parents. Il est nettement plus élevé chez les jeunes issus de familles originaires d'Asie orientale (80\%), d'Afrique du Nord et du Moyen-Orient (74\%), mais relativement moins élevé chez ceux d'Amérique latine et des Caraïbes (58\%).

Par ailleurs, les deux groupes d'étudiants (immigrants et non immigrants) se distinguent entre eux par le type de parcours emprunté et les écarts sont nettement plus prononcés à l'université qu'au CEGEP. Selon l'analyse réalisée à partir de notre échantillon, $56 \%$ des élèves empruntent un parcours linéaire, soit $31 \%$ à l'université et $25 \%$ aux études collégiales. En revanche, $9 \%$ accèdent aux études collégiales à la suite d'un parcours non linéaire combinant les formations professionnelle et générale au secondaire. Alors qu'on peut penser que l'engagement dans parcours linéaire ou non linéaire relève des choix de l'étudiant, les résultats révèlent des différences significatives selon l'origine géographique des parents. Dans l'ensemble, à l'exception de ceux qui viennent d'Amérique latine et des Caraïbes (10\%), les étudiants issus des parents immigrants sont moins enclins à emprunter les parcours non linéaires, comparativement à leurs pairs dont les deux parents sont natifs du Canada (10\%). Ainsi, la proportion de ceux qui s'y inscrivent est relativement faible chez ceux dont les parents viennent des pays de l'Asie orientale (2\%), de même que ceux de l'Asie du Sud-Est et des Îles-du-Pacifique (4\%). À l'inverse, les élèves originaires des familles immigrantes s'inscrivent dans les parcours linéaires jusqu'à l'université dans une proportion relativement plus élevée que leurs pairs dont les parents sont nés au Canada, soit $29 \%$ contre $58 \%$ chez ceux originaires d'Asie orientale, $45 \%$ pour ceux d'Afrique du Nord et du Moyen-Orient, $38 \%$ pour ceux d'Asie du Sud-Est, $36 \%$ pour ceux d'Europe et des pays anglo-saxons et $34 \%$ pour ceux d'Afrique subsaharienne. La situation semble toutefois comparable pour ceux qui s'inscrivent dans un parcours non linéaire aux études collégiales. L'analyse montre qu'il y a relativement peu de différence à ce niveau.

Les analyses multinomiales qui suivent (Tableau 2) visent à examiner si les différences observées selon l'origine géographique des parents sont statistiquement significatives et si elles sont attribuables à cette variable ou à l'effet des antécédents scolaires et caractéristiques sociodémographiques des élèves. Le modèle 1 inclut seulement l'origine géographique des parents de l'élève, en distinguant les immigrants de $1^{\mathrm{e}}$ génération de ceux de $2^{\mathrm{e}}$ génération. Les ré- 
Tableau 2

Coefficients de régression multinomiale selon le type de parcours.

\begin{tabular}{|c|c|c|c|c|c|c|}
\hline & \multicolumn{3}{|c|}{ Modèle 1} & \multicolumn{3}{|c|}{ Modèle 2} \\
\hline & Parcours I & Parcours II & Parcours III & Parcours I & Parcours II & Parcours III \\
\hline & Bêta & Bêta & Bêta & Bêta & Bêta & Bêta \\
\hline \multicolumn{7}{|c|}{ Variable indépendante : origine des parents } \\
\hline $\begin{array}{l}\text { Canada } \\
\text { (référence) }\end{array}$ & 0,000 & 0,000 & 0,000 & 0,000 & 0,000 & 0,000 \\
\hline $\begin{array}{l}\text { Un seul parent } \\
\text { né au Canada }\end{array}$ & $-0,064$ & 0,115 & $-0,418^{* * *}$ & 0,011 & 0,097 & $-0,358^{* *}$ \\
\hline \multicolumn{7}{|l|}{ Asie orientale } \\
\hline $2^{\mathrm{e}}$ génération & $1,345^{*}$ & $2,193^{* * *}$ & 1,000 & $1,658^{* *}$ & $2,249^{* * *}$ & 1,228 \\
\hline $1^{\mathrm{e}}$ génération & 0,152 & $1,060^{* * *}$ & $-2,295^{*}$ & $0,716^{*}$ & $1,634^{* * *}$ & $-1,803$ \\
\hline \multicolumn{7}{|c|}{ Asie du Sud-Est / Îles-du-Pacifique } \\
\hline $2^{\mathrm{e}}$ génération & $0,811^{* * *}$ & $1,270^{* * *}$ & 0,071 & $0,985^{* * *}$ & $1,399^{* * *}$ & 0,144 \\
\hline $1^{\mathrm{e}}$ génération & $-0,359^{*}$ & $1,438^{* *}$ & $-1,420^{* * *}$ & $0,762^{* * *}$ & $1,013^{* * *}$ & $-0,595$ \\
\hline \multicolumn{7}{|c|}{ Afrique du Nord et Moyen-Orient } \\
\hline $2^{\mathrm{e}}$ génération & $0,496^{*}$ & $1,334^{* * *}$ & 0,491 & $0,490^{*}$ & $1,025^{* * *}$ & 0,448 \\
\hline $1^{\mathrm{e}}$ génération & 0,009 & $0,352^{* *}$ & $-0,231$ & $0,541^{* * *}$ & $0,919^{* * *}$ & 0,180 \\
\hline \multicolumn{7}{|c|}{ Afrique subsaharienne } \\
\hline $2^{\mathrm{e}}$ génération & 0,334 & $1,382^{* * *}$ & $1,442^{*}$ & 0,698 & $1,659^{* *}$ & $1,646^{* *}$ \\
\hline $1^{\mathrm{e}}$ génération & $-0,149$ & $-0,305$ & $-1,869^{* *}$ & $0,657^{*}$ & $0,637^{*}$ & $-1,323$ \\
\hline \multicolumn{7}{|c|}{ Europe et pays anglo-saxons } \\
\hline $2^{\mathrm{e}}$ génération & 0,252 & $0,360^{* *}$ & $-0,199$ & $0,441^{* *}$ & $0,560^{* * *}$ & 0,061 \\
\hline $1^{\mathrm{e}}$ génération & $-0,335^{*}$ & 0,178 & $-0,389$ & 0,087 & $0,468^{* *}$ & $-0,402$ \\
\hline \multicolumn{7}{|c|}{ Amérique latine et Caraïbes } \\
\hline $2^{\mathrm{e}}$ génération & $0,344^{* *}$ & $-0,114^{* * *}$ & 0,148 & $0,722^{* * *}$ & 0,238 & $0,379^{*}$ \\
\hline $1^{\mathrm{e}}$ génération & $-0,588^{* * *}$ & $-1,228^{* * *}$ & $0,171^{* *}$ & $0,276^{*}$ & $-0,140$ & 0,148 \\
\hline \multicolumn{7}{|l|}{ Variables de contrôle } \\
\hline \multicolumn{7}{|c|}{ Antécédents scolaires } \\
\hline $\begin{array}{l}\text { A connu des } \\
\text { difficultés } \\
\text { scolaires (oui) }\end{array}$ & - & - & - & $-1,374^{* * *}$ & $-2,319^{* * *}$ & $-0,966^{* * *}$ \\
\hline $\begin{array}{l}\text { Age d'entrée } \\
\text { en } 1^{\mathrm{e}} \\
\text { secondaire }\end{array}$ & - & - & - & $-0,859^{* * *}$ & $-1,353^{* * *}$ & $-0,472^{* * *}$ \\
\hline
\end{tabular}

Continuer... 
Tableau 2

Suite.

\begin{tabular}{l|c|c|c|c|c|c}
\hline & \multicolumn{3}{|c|}{ Modèle 1 } & \multicolumn{3}{c}{ Modèle 2 } \\
\cline { 2 - 7 } & Parcours I & Parcours II & Parcours III & Parcours I & Parcours II & Parcours III \\
\cline { 2 - 7 } & Bêta & Bêta & Bêta & Bêta & Bêta & Bêta \\
\hline $\begin{array}{l}\text { Type } \\
\text { d'établissement } \\
\begin{array}{l}\text { secondaire } \\
\text { (privé) }\end{array}\end{array}$ & - & - & - & $0,901^{* * *}$ & $1,517^{* * *}$ & $0,620^{* * *}$ \\
\hline
\end{tabular}

\begin{tabular}{l|c|c|c|c|c|c}
\hline \multicolumn{2}{l}{ Caractéristiques sociodémographiques } & - & - & $0,829^{* * *}$ & $0,890^{* * *}$ & $0,397^{*}$ \\
\hline $\begin{array}{l}\text { Revenu annuel } \\
\text { médian / } \\
\text { ménage (log) }\end{array}$ & - & - & - & $0,010^{* * *}$ & $0,026^{* * *}$ & $-0,005^{*}$ \\
\hline $\begin{array}{l}\text { \% diplômés } \\
\text { universitaires }\end{array}$ & - & - & - & $-0,608^{* * *}$ & $-0,826^{* * *}$ & $-0,224^{* * *}$ \\
\hline Sexe (homme) & - & - & - & $-0,134^{* * *}$ & $0,112^{* *}$ & $0,437^{* * *}$ \\
\hline $\begin{array}{l}\text { Cohorte } \\
\text { 2002-2003 }\end{array}$ & - & - & & 0,123 & & \\
\hline Pseudo-R & 0,010 & & & $6082,20^{* * *}$ & & \\
\hline $\mathrm{c}_{\mathrm{RV}}^{2}$ & $391,19^{* * *}$ & & & 60 & & \\
\hline $\mathrm{D} . \mathrm{L}$. & 19688 & & & 19097 & & \\
\hline $\mathrm{N}$ & & & & &
\end{tabular}

Note:

- parcours I : linéaire secondaire $\Rightarrow C E G E P$; parcours $I I$ : linéaire secondaire $\Rightarrow C E G E P \Rightarrow$ université ; parcours III : non linéaire secondaire $\Rightarrow C E G E P$.

- ${ }^{*}$ ignificatif au seuil de 0,05; ${ }^{* *}$ significatif au seuil de 0,01; *** significatif au seuil de 0,001.

sultats précisent ceux de l'analyse descriptive (Tableau 1), révélant la présence de différences significatives pour les parcours linéaires et, dans une moindre mesure, pour les parcours non linéaires. Les immigrants sont davantage enclins à emprunter les parcours linéaires; à l'inverse, ils sont moins susceptibles de s'engager dans les parcours non linéaires.

En ce qui concerne les parcours linéaires, une analyse attentive des résultats permet de dégager trois constats principaux. Premièrement, les différences sont nettement plus élevées et significatives à l'université qu'au CEGEP. Deuxièmement, la situation est fortement variable parmi les immigrants. Ainsi, par rapport au non-immigrants, la probabilité de suivre ce parcours est neuf fois plus élevée $(\operatorname{Exp}(2,193)=8,962)$ chez les immigrants de $2^{\mathrm{e}}$ génération originaires d'Asie orientale et presque quatre fois plus élevée chez ceux originaires d'Asie du Sud-Est et des Îles du Pacifique $(\operatorname{Epx}(1,270)=3,561)$, d'Afrique du Nord et du Maghreb et du Moyen-Orient $(\operatorname{Exp}(1,334)=3,796)$ et d'Afrique subsaharienne $(\operatorname{Exp}(1,382)=$ 
3,982). À noter cependant que les immigrants originaires d'Amérique latine et des Caraïbes font exception et sont moins susceptibles de suivre les parcours linéaires à l'université, comparativement aux non-immigrants $(\operatorname{Exp}(-0,114=0,892)$. Troisièmement, les différences significatives se rapportent principalement aux immigrants de $2^{\mathrm{e}}$ génération, alors qu'elles sont plutôt modérées, voire nulles pour ceux de $1^{\text {e }}$ génération dans le cas du CEGEP.

En ce qui a trait aux parcours non linéaires, la situation est quelque peu inversée, comme souligné précédemment : les immigrants sont moins susceptibles de suivre ces parcours. À cet égard, l'analyse permet aussi de dégager deux observations: les différences significatives observées sont encore une fois très variables selon l'origine géographique des parents de l'élève, mais se rapportent cette fois-ci aux immigrants de $1^{\mathrm{e}}$ génération, alors que la situation est plutôt comparable entre ceux de $2^{\mathrm{e}}$ génération et leurs pairs de $3^{\mathrm{e}}$ génération (les non-immigrants).

Le modèle 2 tient compte des variables de contrôle en lien avec les caractéristiques du parcours au secondaire (la présence d'un code de difficulté dans le dossier scolaire de l'élève, le type d'école fréquenté et l'âge d'entrée en $1^{\mathrm{e}}$ année du secondaire), ainsi que de trois variables sociodémographiques (le genre, le revenu médian des ménages et le pourcentage de diplômés d'université résidant dans la région habitée par les parents selon le recensement de 2006). Les résultats révèlent que ces variables exercent une influence significative sur l'accès aux études supérieures et l'usage des différents parcours. Les élèves ayant connu un retard ou des difficultés scolaires au secondaire ont moins de chances d'accéder aux études supérieures, que ce soit par la voie d'un parcours linéaire ou non linéaire. Ici, encore une fois, les différences observées sont nettement plus élevées au niveau des parcours linéaires à l'université.

La prise en compte de ces deux catégories de variables modifie cependant considérablement l'influence de l'origine géographique des parents de l'élève, révélant qu'à caractéristiques scolaires et sociodémographiques comparables, les immigrants sont nettement plus susceptibles de poursuivre les études supérieures à l'université comme au CEGEP par la voie des parcours linéaires, qu'ils soient de $1^{\mathrm{e}}$ ou de $2^{\mathrm{e}}$ génération. Par contre, on observe relativement moins de différences au sujet des parcours non linéaires. Une analyse supplémentaire révèle toutefois que cette modification de résultats est davantage attribuable aux caractéristiques scolaires qu'aux caractéristiques sociodémographiques de l'élève, ce qui appuie l'hypothèse que les parcours scolaires des élèves immigrants aux études supérieures sont fortement modulés par leurs antécédents scolaires au secondaire, comme THIESSEN (2009) l'avait souligné dans une étude pancanadienne.

En somme, l'accès aux études supérieures et l'usage des parcours scolaires linéaires varient significativement entre les immigrants et les non-immi- 
grants, de même qu'entre les immigrants de $1^{\mathrm{e}}$ et de $2^{\mathrm{e}}$ génération. Lorsqu'on tient compte de leurs antécédents scolaires, les différences observées tendent même à augmenter. En revanche, la morphologie des parcours scolaires des immigrants, qu'ils soient de $1^{\mathrm{e}}$ ou de $2^{\mathrm{e}}$ génération, tend à devenir plus ou moins similaire : bien que l'ampleur soit variable, ils ont tous en commun la probabilité d'accès aux études supérieures et de choix des parcours linéaires plus élevée que leurs pairs non immigrants.

\section{Discussion et conclusion}

L'objectif de cette étude était de décrire et de comprendre la morphologie des parcours des jeunes Canadiens issus des familles d'origine immigrante vers et dans l'enseignement supérieur, ainsi que les facteurs qui contribuent à leur structuration. Les résultats obtenus à partir des données du ministère de l'Éducation de la province du Québec permettent de dégager les constats suivants. Premièrement, ces jeunes poursuivent les études supérieures par la voie des parcours linéaires dans une proportion plus élevée que leurs pairs dont les parents sont nés au Canada. Deuxièmement, les différences sont nettement plus élevées à l'université qu'au CEGEP: les étudiants d'origine immigrante s'inscrivent davantage dans les parcours linéaires jusqu'à l'université, à l'exception de ceux d'origine latino-américaine et caribéenne. Troisièmement, au sein des immigrants, il existe des différences significatives élevées: l'accès aux études supérieures et l'usage des parcours scolaires linéaires varient selon l'origine géographique des parents et selon que l'étudiant est un immigrant de $1^{\mathrm{e}}$ ou de $2^{\mathrm{e}}$ génération. En particulier, les immigrants de $2^{e}$ génération originaires d'Asie orientale et dans une moindre mesure, ceux d'Afrique du Nord et du MoyenOrient sont nettement plus susceptibles que les autres d'accéder aux études supérieures et de suivre les parcours linéaires jusqu'à l'université. À l'inverse, les immigrants d'origine latino-américaine et caribéenne, qu'ils soient de $1^{\mathrm{e}}$ ou de $2^{\mathrm{e}}$ génération, sont les moins susceptibles d'accéder aux études supérieures par la voie des parcours linéaires. En somme, en dépit de l'ampleur des différences d'accès aux études supérieures, la morphologie des parcours scolaires tend à être similaire entre les immigrants lorsqu'on tient compte des antécédents scolaires au secondaire: ils ont en commun la tendance à choisir davantage les parcours linéaires lorsqu' ils ont un bon dossier scolaire.

Les résultats de cet article tendent à corroborer ainsi ceux des études antérieures récentes qui soulignent, à l'échelle canadienne, la participation accrue des immigrants aux études supérieures (FINNIE \& MUELLER, 2010), mais également des différences significatives entre les jeunes issus de l'immigration euxmêmes selon l'origine de leurs parents (KAMANZI et al., 2016; THIESSEN, 2009). Alors que les études à l'échelle internationale reconnaissent les ségrégations 
dont les immigrants font l'objet (LEVELS ; DRONKERS ; KRAAYKAMP, 2008; SÖHN \& ÖZCAN, 2006), trois explications à la fois complémentaires et contradictoires ont été avancées pour expliquer l'exception canadienne.

La première renvoie aux aspirations scolaires élevées des jeunes issus de l'immigration (KAMANZI, 2013; THIESSEN, 2009), mais aussi et surtout à celles de leurs parents (TAYLOR \& KRAHN, 2005) qui consacrent beaucoup de ressources, souvent au prix de grands sacrifices qui dépassent leurs moyens, pour scolariser leurs enfants jusqu'aux études supérieures (ANISEF ; BROWN ; SWEET , 2011). La deuxième piste serait que les aspirations des jeunes immigrants canadiens se conjuguent à la qualité des parcours scolaires au primaire et au secondaire. Alors que des études de comparaison internationale témoignent que, dans plusieurs pays occidentaux, les élèves d'origine immigrante se caractérisent par des performances scolaires faibles et des parcours scolaires difficiles (DRONKERS \& VAN DER VELDEN, 2013), qui réduisent ainsi leurs chances d'accès à l'enseignement supérieur, le Canada ferait partie des exceptions. De manière générale, les élèves issus des familles immigrantes et leurs pairs issus des familles natives ont des résultats scolaires comparables (HOCHSCHILD \& CROPPER, 2010). La troisième piste renvoie au contexte social général (SCHNEIDER \& CRUL, 2012), mais surtout au caractère des politiques canadiennes d'immigration qui se distinguent par la volonté et des mesures d'intégration socioéconomique des immigrants (BLOEMRAAD, 2012). Cette piste prend deux directions. La première est que non seulement les immigrants sont scolarisés dans les mêmes établissements que les autres jeunes Canadiens, mais des ressources supplémentaires sont également allouées au soutien à leur intégration, dans le cas des élèves nés à l'étranger (SHAKYA et al., 2010). La deuxième, qui complète la première, tout en la nuançant, tient aux caractéristiques des parents d'élèves et aux politiques de sélection auxquelles sont soumis les immigrants. Tout en reconnaissant que les politiques d'immigration favorisent la réussite scolaire des jeunes issus de l'immigration, certains auteurs comme Boyd (2002), Rothon, Heath \& Lessard-Phillips (2009), Hochschild \& Cropper (2010) et, plus récemment, Mc Andrew (2015), soutiennent que cette réussite est en grande partie attribuable aux caractéristiques des parents immigrants. Des recherches indiquent que le Canada fait partie des pays qui sélectionnent davantage les immigrants en fonction de leur capital scolaire et économique (LOWELL, 2005).

Au terme de cette étude, deux limites méthodologiques associées aux contraintes de la base de données utilisée doivent cependant être soulignées. La première tient au regroupement des sujets en fonction des régions géographiques d'origine des parents. Tout en faisant partie d'un même espace géographique, les pays d'origine des immigrants canadiens et, par conséquent, ces immigrants euxmêmes, se différencient entre eux par plusieurs aspects, notamment valeurs culturelles de leurs communautés, l'histoire de leurs pays et leur processus d'immigration (LEVELS ; DRONKERS ; KRAAYKAMP, 2008). La deuxième est d'avoir 
restreint l'analyse à la région de Montréal en raison des effectifs de sujets faibles dans les autres régions. La troisième limite tient au biais d'attrition des décrocheurs qui sont relativement plus nombreux dans les communautés noires issues d'Afrique noire et des Caraïbes, comme le soulignent les travaux récents de Mc Andrew (2015), alors que l'accès aux études supérieures est conditionnel à la persévérance et à l'obtention du diplôme d'études secondaires. Dans la même veine, la base de données utilisée ne contient pas d'information sur les résultats scolaires qui conditionnent également les parcours et l'accès aux études supérieures.

En dépit de ces limites, l'étude est arrivée à des résultats intéressants au plan social et scientifique et ouvre de nouvelles perspectives de recherche. Parmi celles-ci, on peut mentionner la compréhension des parcours scolaires des élèves fréquentant des établissements situés en milieux défavorisés et pluriethniques qui demeurent un lieu d'(auto)exclusion sociale et d'inégalités scolaires au Québec et à Montréal en particulier (VIDAL, 2017).

\section{Notes}

1. Collège d'enseignement général et professionnel (College of General and Vocational Education), premier niveau de l'enseignement supérieur au Québec.

2. Les adultes ou les jeunes qui sont nés et ont grandi dans un autre pays que celui où ils ont immigré et vivent.

3. Les adultes ou les jeunes nés dans le pays d'immigration de leurs parents.

\section{Références}

ALBA, R.; NEE, V. Rethinking Assimilation Theory for a New Era of Immigration. The International Migration Review, v. 31, n. 4, p. 826-874, 1997. https://doi. org/10.2307/2547416

ALBA, R.; SILBERMAN, R. Decolonization Immigrations and the Social Origins of the Second Generation: The Case of North Africans in France. International Migration Review, v. 36, n. 4, p. 1169-1193, 2002. https://doi.org/10.1111/j.1747-7379.2002.tb00122.x

ALLISON, P. D. Logistic Regression. Using the SAS System. Theory and Application. Cary: SAS Institute Inc., 2003.

ANISEF, P. S.; BROWN, R.; SWEET, R. Post-secondary Pathway Choices of Immigrant and Native-born Youth in Toronto. Thèmes canadiens/Canadian Issues, p. 42-48, 2011.

BANKSTON, III C. L. Context and Contradictory Consequences. Sociology of Education, v. 77, n. 2, p. 176-179, 2004. https://doi.org/10.1177/003804070407700205

BAUM, S.; FLORES, S. M. Higher Education and Children in Immigrant Families. The Future of Children, v. 21, n. 1, p. 171-193, 2011. 
BELOT, M.; EDERVEEN, S. Cultural Barriers in Migration between OECD Countries. Journal of Population Economics, v. 25, n. 3, p. 1077-1105, 2012. https://doi. org/10.1007/s00148-011-0356-x

BERRY, J. W.; KALIN, R. Multicultural and Ethnic Attitudes in Canada: An Overview of the 1991 National Survey. Canadian Journal of Behavioural Science, v. 27, n. 3, p. 301-320, 1995. http://psycnet.apa.org/doi/10.1037/0008-400X.27.3.301

BLOEMRAAD, I. Understanding “Canadian Exceptionalism” in Immigration and Pluralism Policy. Washington, D.C.: Migration Policy Institute, 2012.

BORJAS, G. Making It in America: Social Mobility in the Immigrant Population. The Future of Children, v. 16, n. 2, p. 55-71, 2006.

BOURDIEU, P. L'école conservatrice. Les inégalités devant l'école et devant la culture. Revue Française de Sociologie, v. 7, n. 3, p. 325-347, 1966. https://doi.org/10.2307/3319132

BOYD, M. Educational Attainments of Immigrant Offspring: Success or Segmented Assimilation? International Immigration Review, v. 36, n. 4, p. 1037-1060, 2002.

BRINBAUM, Y.; LUTZ, A. Examining Educational Inequalities in two National Systems: a Comparison of the North African Second Generation in France and the Mexican Second Generation in the United States. Journal of Ethnic and Migration Studies, v. 43, n. 15, p. 2591-2615, 2017. https://doi.org/10.1080/1369183X.2017.1290522

BUCHMANN, C.; PARRADO, E. A. Educational Achievement of Immigrant-origin and Native Students: A comparative Analysis Informed by Institutional Theory. In: BAKER, D. P.; WISEMAN, A. W. (orgs.) The Impact of Comparative Education Research on Institutional Theory. Bingley: Emerald Group Publishing Limited, 2006. p. 335-366.

CRUL, M. et al. School careers of second-generation youth in Europe: Which education systems provide the best chances for success? In: CRUL, M.; SCHNEIDER, J.; LELIE, F. (orgs.). The European Second Generation Compared. Does the Integration Context Matter? Amsterdam: Amsterdam University Press, 2012. p. 110-165.

DRONKERS, J.; VAN DER VELDEN, R. Positive but also Negative Effects of Ethnic Diversity in Schools on Educational Performance? An Empirical Test Using PISA Data. In: WINDZIO, M. (org.). Integration and Inequality in Educational Institutions. Dordrecht / Heidelberg / New York / London: Springer, 2013. p. 71-98.

DUSTMANN, C.; FRATTINI, T.; LANZARA, G. Educational achievement of second generation of immigrant: an international comparison. London: Centre for Research and Analysis of Migration, Department of Economics, 2011.

ELDERING, L.; KNORTH, E. J. Marginalization of Immigrant Youth and Risk Factors in Their Everyday Lives: The European Experience. Child \& Youth Care Forum, v. 27, n. 3, p. 153-169, 1998. https://doi.org/10.1007/BF02589561

FINNIE, R.; MUELLER, R. E. They Came, they Saw, they Enrolled: Access to Post-secondary Education by the Children of Canadian Immigrants. In: FINNIE, R.; FRENETTE, M.; MUELLER, R. E.; SWEETMAN, A. (orgs.). Pursuing higher education in Canada. Economic, social, and policy dimensions. Kingston: Queen's University Press, 2010. p. 192-216. 
GREENMAN, E.; XIE, Y. Is assimilation Theory Dead? The Effect of Assimilation on Adolescent Well-being. Social Science Research, v. 37, v. 1, p. 109-137, 2008. https://dx.doi.org/10.1016\%2Fj.ssresearch.2007.07.003

GRIGA, D.; HADJAR, A. Migrant Background and Higher Education Participation in Europe: The Effect of the Educational Systems. European Sociological Review, v. 30, n. 3, p. 275-286, 2013. https://doi.org/10.1093/esr/jct031

HAO, L.; BONSTEAD-BRUNS, M. Parent-Child Differences in Educational Expectations and the Academic Achievement of Immigrant and Native Students Sociology of Education, v. 71, n. 3, p. 175-198, 1998. https://doi.org/10.2307/2673201

HENRY, F; TATOR, C. Racism in the Canadian University: Demanding Social Justice, Inclusion, and Equity. Toronto: University of Toronto Press, 2009.

HOCHSCHILD, J. L.; CROPPER, P. Immigration Regimes and Schooling Regimes: Which Countries Promote Successful Immigrant Incorporation? Theory and Research in Education, v. 8, n. 1, p. 21-61, 2010. https://doi.org/10.1177\%2F1477878509356342

HOELSCHER, M.; HAYWARD, G.; ERTL, H.; DUNBAR-GODDET, H. The Transition from Vocational Education and Training to Higher Education: a Successful Pathway? Research Papers in Education, v. 23, n. 2, p. 139-151, 2008. https://doi. org/10.1080/02671520802048679

ICHOU, M. 'Who they Were there: Immigrants' Educational Selectivity and their Children's Educational Attainment. European Sociological Review, v. 30, n. 6, p. 750 765, 2014. https://doi.org/10.1093/esr/jcu071

KAMANZI, P. C. La mobilisation pour les études et les étudiants immigrants au Québec. In: CHENARD, P.; DORAY, P.; DUSSAULT, E.-L.; RINGUETTE, M. (orgs.). L'accessibilité aux études postsecondaires: un projet inachevé. Montréal: Presses de l'Université du Québec, 2013. p. 305-319.

KAMANZI, P. C.; BASTIEN, N.; DORAY, P.; MAGNAN, M.-O. Immigration et cheminements scolaires aux études supérieures au Canada : qui y va et quand? Une analyse à partir du modèle de risque proportionnel de Cox. Canadian Journal of Higher Education/Revue canadienne d'enseignement supérieur, v. 46, n. 2, p. 225-248, 2016.

KAMANZI, C.; ZHANG, X. Y.; DEBLOIS, L.; DENIGER, M.A. L'influence du capital social sur la formation du capital humain chez les élèves résilients de milieux socioéconomiques défavorisés. Revue des sciences de l'éducation, v. 33, n. 1, p. 127 145, 2007. http://dx.doi.org/10.7202/016192ar

LEVELS, M.; DRONKERS, J.; KRAAYKAMP, G. Immigrant Children's Educational Achievement in Western Countries: Origin, Destination, and Community Effects on Mathematical Performance. American Sociological Review, v. 73, n. 5, p. 835-853, 2008. https://doi.org/10.1177\%2F000312240807300507

LOWELL, B. L. Policies and Regulations for Managing Skilled International Migration for Work. New York: United Nations, Population Division Department of Economic and Social Affairs United Nations Secretariat, 2005. 
MACLEAN, R.; PAVLOVA, M. Vocationalization of Secondary and Higher Education: Pathways to the World of Work. In: UNITED NATIONS EDUCATIONAL, SCIENTIFIC AND CULTURAL ORGANIZATION. Revisiting Global Trends in TVET: Reflections on Theory and Practice. Bonn: Unesco, 2013.

MC ANDREW, M. La réussite éducative des élèves issus de l'immigration: dix ans de recherches et d'intervention. Montréal: Presses de l'Université de Montréal, 2015.

NIELSEN, H. S.; ROSHOLM, M.; SMITH, M.; HUSTED, L. The School-towork Transition of 2nd Generation Immigrants in Denmark. Journal of Population Economics, v. 16, n. 4, p. 755-786, 2003.

PORTES, A.; FERNÁNDEZ-KELLY, P.; HALLER, W. Segmented Assimilation on the Ground: The New Second Generation in early Adulthood. Ethnic and Racial Studies, v. 28, n. 6, p. 1000-1040, 2005. https://doi.org/10.1080/01419870500224117

PORTES, A.; ZHOU, M. The new Second Generation: Segmented Assimilation and its Variants. Annals of the American Academy of Political and Social Science, v. 530, p. 74-96, 1993.

ROTHON, C.; HEATH, A.; LESSARD-PHILIPS, L. The Educational Attainments of the "Second Generation": A Comparative study of Britain, Canada, and the United States. Teachers College Record, v. 111, n. 6, p. 1404-1443, 2009.

SCHNEIDER, J.; CRUL, M. Comparative Integration Context Theory: Participation and Belonging in Diverse European Cities. In: CRUL, M.; SCHNEIDER, J.; LELIE, F. (orgs.). The European Second Generation Compared Does the Integration Context Matter? Amsterdam: Amsterdam University Press, 2012. p. 19-37.

SHAKYA, Y.B. et al. Aspirations for Higher Education among Newcomer Refugee Youth in Toronto: Expectations, Challenges, and Strategies. Refugee, v. 27, n. 2, p. 65-78, 2010.

SILBERMAN, R.; ALBA, R.; FOURNIER, I. Discrimination in Labour Market against the Second Generation. Ethnic and Racial Studies, v. 3, n. 1, p. 1-27, 2006.

SÖHN, J.; ÖZCAN, V. The Educational Attainment of Turkish. Turkish Studies, v. 7, n. 1, p. 101-124, 2006. https://doi.org/10.1080/14683840500520626

TAYLOR, A.; KRAHN, H. Aiming high: Educational Aspirations of Visible Minority Immigrant Youth. Canadian Social Trends, v. 79, p. 8-12, 2005.

TELTEMANN, J.; SCHUNCK, R. Education Systems, School Segregation, and Second-generation Immigrants' Educational Success: Evidence from a Country-fixed Effects Approach Using three Waves of PISA. International Journal of Comparative Sociology, v. 57, n. 6, p. 401-424, 2016. https://doi. org/10.1177\%2F0020715216687348

THIESSEN, V. The Pursuit of Postsecondary Education: A Comparison of First Nations, African, Asian, and European Canadian Youth. Canadian Review of Sociology, v. 46, n. 1, p. 5-37, 2009. https://doi.org/10.1111/j.1755-618X.2009.01201.x 
VIDAL, M. Comprendre pour mieux intervenir : la production du capital social dans une école secondaire en milieu défavorisé et multiethnique. 2017. Thèse (Doctorat) Université de Montréal, Montréal, 2017.

XIE, Y.; GREENMAN, E. The Social Context of Assimilation: Testing Implications of Segmented Assimilation Theory. Social Science Research, v. 40, n. 3, p. 965-984, 2011. https://dx.doi.org/10.1016\%2Fj.ssresearch.2011.01.004

ZHOU, M. Segmented Assimilation: Issues, Controversies, and Recent Research on the new Second Generation. International Migration Review, v. 31, n. 4, p. 975-1008, 1997. https://doi.org/10.2307/2547421

Reçu le 18 juin 2017.

Accepté le 4 septembre 2018. 